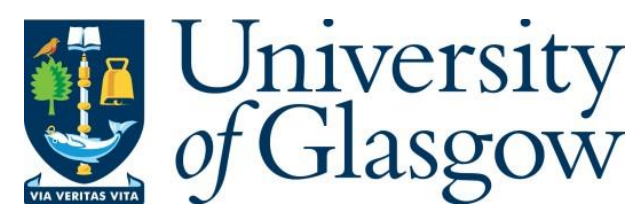

Wisbey, D. S. et al. (2019) Dielectric loss of boron-based dielectrics on niobium resonators. Journal of Low Temperature Physics, 195(5-6), pp. 474-486.

There may be differences between this version and the published version. You are advised to consult the publisher's version if you wish to cite from it.

http://eprints.gla.ac.uk/187357/

Deposited on: 28 May 2019

Enlighten - Research publications by members of the University of Glasgow http://eprints.gla.ac.uk 


\title{
Dielectric Loss of Boron-Based Dielectrics on Niobium Resonators
}

\author{
David S. Wisbey - Michael R. Vissers - Jiansong Gao - Jeff S Kline • \\ Martin O. Sandberg • Martin P. Weides - M. M. Paquette - S. Karki • \\ Jacob Brewster • Dheyaa Alameri - Irma Kuljanishvili • Anthony N. \\ Caruso - Dave P. Pappas
}

Received: date / Accepted: date

\begin{abstract}
Advanced solid state quantum bits (qubits) are likely to require a variety of dielectrics for wiring crossovers, substrates, and Josephson junctions. Microwa superconducting resonators are an excellent tool for measuring the internal dielectric loss of materials. We report the dielectric loss of boron-based dielectric films using a microwave coplanar waveguide (CPW) resonator with heterostructure geometry. Power-dependent internal quality factors of magnetron-sputtered boron carbide $\left(\mathrm{B}_{4} \mathrm{C}\right)$ and boron nitride $(\mathrm{BN})$ were measured and

This work was funded by the U.S. government, Office of the Director of National Intelligence (ODNI), Intelligence Advanced Research Projects Activity (IARPA), and the NIST Quantum-based Sensors and Measurements Initiative. All statements of fact, opinion, or conclusions contained herein are those of the authors and should not be construed as representing the official views or policies of ODNI or IARPA. UMKC acknowledges financial support from the Defense Threat Reduction Agency under grant no. HDTRA1-10-10092 .
\end{abstract}

D. Wisbey, J. Brewster, D. Alameri, I. Kuljanishvili

Saint Louis University, Department of Physics, St. Louis Missouri, USA

Tel.: 314-882-4312 Fax: 314-977-8384

E-mail: david.wisbey@slu.edu ORCID ID:

https://orcid.org/0000-0001-6376-6052

M. Vissers, J. Gao, J. Kline, D. P. Pappas

National Institute of Standards and Technology, Boulder Colorado, USA

M. Sandberg

IBM, Armonk, New York, NY, USA

M. Weides

School of Engineering, University of Glasgow, Glasgow G12 8QQ, UK

M. Paquette, S. Karki, A. N. Caruso

University of Missouri-Kansas City, Department of Physics and Astronomy, Kansas City Missouri, USA are compared to silicon oxide $\left(\mathrm{SiO}_{2}\right)$, a common material used in wiring crossovers. The internal dielectric loss due to two-level systems for $\mathrm{B}_{4} \mathrm{C}$ and $\mathrm{BN}$ is less than silicon dioxide $\left(\mathrm{SiO}_{2}\right)$, which demonstrates the existence of low loss sputtered materials. We also found that niobium $(\mathrm{Nb}) \mathrm{CPW}$ resonators suffer a decrease in internal quality factor after deposition of $\mathrm{B}_{4} \mathrm{C}$ at temperatures above $150^{\circ} \mathrm{C}$. This result is consistent with the idea that the oxidation of the surface of the superconducting metal can contribute to loss in a device.

Keywords Superconducting resonator · low temperature loss $\cdot$ two-level systems $\cdot$ boron nitride $\cdot$ boron carbide

\section{Introduction}

Evaluating the loss in dielectric materials at very low temperatures is important for the continued advance of superconducting circuits used in quantum information processing (QIP). Currently, materials used in solid state heterostructure devices are a limiting factor, preventing the realization of a scalable quantum computer. Microwave resonators are an excellent tool for studying the various properties, most importantly the loss, of materials to be used for fabricating qubits. This is because they are both an intrinsic component and a good test bed for loss measurements [1].

The dielectric loss of boron carbide $\left(\mathrm{B}_{4} \mathrm{C}\right)$ and boron nitride $(\mathrm{BN})$ was measured using microwave resonators. Boron nitride and boron carbide are some of the hardest materials in the world after diamond and cubic $\mathrm{BC}_{2} \mathrm{~N}$ [2]. This hardness is associated with a high electron bond strength, a lower amount of unpaired electrons, a strong tendency for covalent bond formation, and a lack of dangling bonds. Dangling bonds at the surfaces 
of metal electrodes and dielectric interfaces have been associated with decoherence and increased $1 / f$ noise in superconducting microwave devices $[3,4]$. Boron nitride is a particularly interesting material because it has tunable properties such as band gap, stoichiometry, and bond strength [5].

Microwave loss in a dielectric is referred to as $\delta_{i}=$ $1 / Q_{i}$ where $Q_{i}$ is the internal quality factor. The internal quality factor is defined as the energy stored in the resonator divided by energy lost to internal sources of the device. Internal sources of loss can include a variety of things; however, at low temperatures and low microwave power, it is often dominated by two-level systems [6]. A previously established standard for testing and quantifying the microwave loss of new materials in circuits for quantum information processing (QIP) [7$10]$ was used.

This paper shows that $\mathrm{B}_{4} \mathrm{C}$ and $\mathrm{BN}$ have a lower dielectric loss than $\mathrm{SiO}_{2}$ grown with electron cyclotron resonance (ECR). Wiring crossovers made of $\mathrm{SiO}_{2}$ were commonly used previously. While the measured loss is larger than that obtained for the lowest loss deposited dielectric a-Si:H [7], boron-based dielectric can be sputterdeposited at room temperature, which is advantageous. The material a-Si:H usually requires growth at an elevated temperature of around $250^{\circ} \mathrm{C}$ [11]. Dielectrics that require heating the sample can cause adverse effects, e.g., hydrogen or silicon may get incorporated into the superconductor or the crystal structure of the underlying layers may change. We also show that heating a niobium resonator causes the quality factor of the resonator to drop and the resonance frequency to change.

\section{Experimental}

\subsection{Microwave Measurement Setup}

An adiabatic demagnetization refrigerator (ADR) was used to conduct the low temperature measurements. The system had a base temperature of less than $80 \mathrm{mK}$ [12], as measured using a $\mathrm{RuO}_{2}$ sensor attached to the sample holder. The experimental setup used to make $\mathrm{RF}$ measurements is shown in Fig. 1. Measurements consisted of transmission $S_{21}$ using a $1 \mathrm{GHz}-20 \mathrm{GHz}$ vector network analyzer (VNA). The input microwave signal was attenuated by $70 \mathrm{~dB}$ before reaching the device. The various attenuators are shown at the different temperature stages in Fig 1. The signal passed through four DC blocks, two at the $500 \mathrm{mK}$ stage and two at the $50 \mathrm{mK}$ stage. The transmitted signal from the sample was sent to the $500 \mathrm{mK}$ stage where it passed through a bias tee, to cool the center conductor of the coaxial cable, and a directional circulator, and then to a high

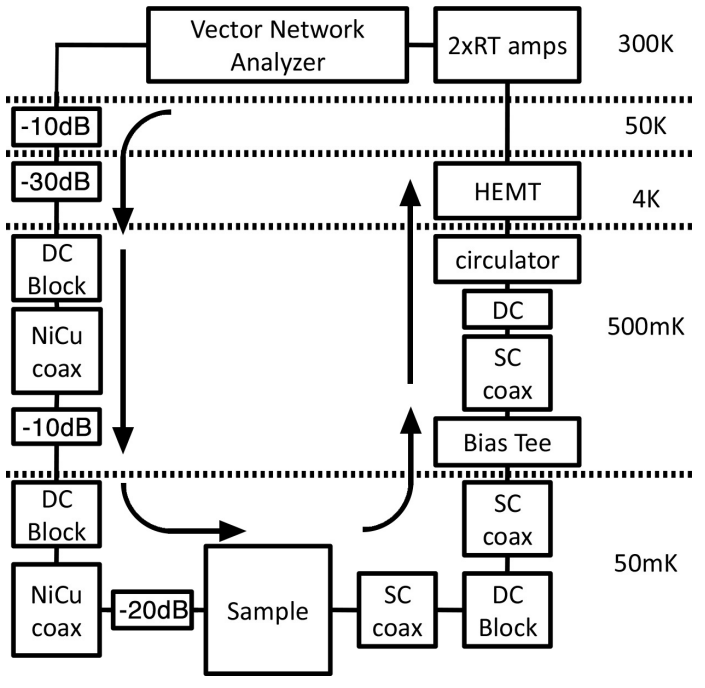

Fig. 1 Schematic of the microwave measurement setup. The arrows indicate the direction of the microwave signal flow. The sample was at $80 \mathrm{mK}$ while the bias $\mathrm{T}$ and directional circulator were at the $500 \mathrm{mK}$ stage during measurement. There were four DC blocks, two at the $50 \mathrm{mK}$ stage, and two at the $500 \mathrm{mK}$ stage. The HEMT amplifier was at $4 \mathrm{~K}$. The attenuators were distributed over the various temperature stages as shown, with two amplifiers at room temperature.

electron mobility transistor (HEMT) amplifier at the 4 $\mathrm{K}$ stage. The HEMT amplifier gave about $40 \mathrm{~dB}$ amplification over the bandwidth used.

Another $30 \mathrm{~dB}$ amplification was achieved with external room temperature amplifiers. Because the quarter wave CPW resonators are coupled to a continuous CPW feedline, we measure a dip in the transmitted power at the resonance frequency of each resonator. This paper follows a well demonstrated technique [610,12 ] to measure the loss tangent of dielectric materials. Coplanar waveguides are advantageous for materials studies because they are well understood, straightforward to analyze, and the loss, $1 / Q_{i}$, is additive, and thus easy to separate into different components $[7,15]$. Measurements of the TLS loss were determined using both the power and temperature dependence of resonance $[16]$.

\subsection{Fabrication and Resonator Design}

The low temperature dielectric loss of our samples was measured using quarter wave coplanar waveguide $(\mathrm{CPW})$ resonators. The $\mathrm{SiO}_{x}$ and $\mathrm{B}_{4} \mathrm{C}$ data were measured using a CPW resonator composed of $200 \mathrm{~nm}$ of niobium $(\mathrm{Nb})$ on a sapphire substrate. Devices with $\mathrm{BN}$ were measured with a $200 \mathrm{~nm} \mathrm{Nb}$ resonator on an intrinsic $\mathrm{Si}(100)$ wafer. Sapphire was chosen for the $\mathrm{B}_{4} \mathrm{C}$ devices so that the dielectric could be deposited at high temperature without affecting the substrate. This allowed $\mathrm{B}_{4} \mathrm{C}$ 


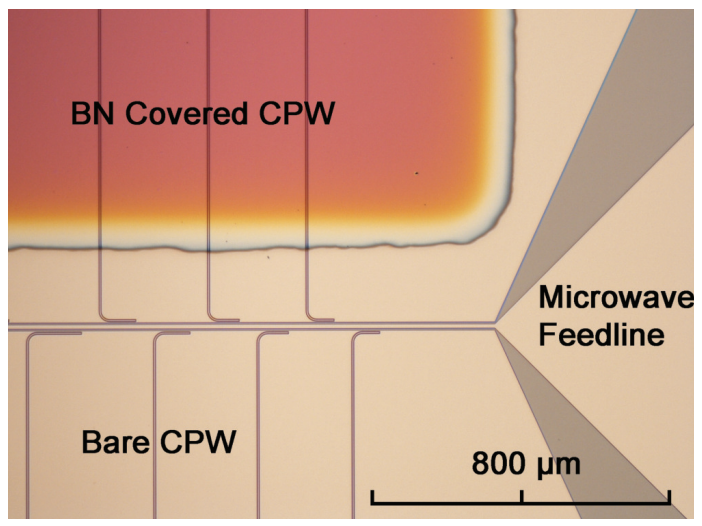

Fig. 2 Frequency multiplexed niobium quarter wave coplanar waveguide (CPW) resonators covered with $300 \mathrm{~nm}$ of deposited BN.

samples grown at high and low temperatures to be compared. The niobium CPW resonators were capacitively coupled to a continuous CPW feedline for transmission measurements. They were etched using $\mathrm{SF}_{6}$ at low $\mathrm{RF}$ power [12]. The samples were grown on $76 \mathrm{~mm}$ (3”) wafers of either c-plane sapphire or intrinsic $\mathrm{Si}(100)$ with a resistivity of $17 \mathrm{k} \Omega-\mathrm{cm}$. The sapphire substrates were annealed in a tube furnace for $20 \mathrm{~h}$ at $1100^{\circ} \mathrm{C}$ in an oxygen atmosphere in situ to prepare the surface. After annealing the substrates, the surface is much smoother and more crystalline, as described previously [13]. For the Si substrates, prior to deposition, the native oxide was removed using $\mathrm{HF}$ as described previously [12]. A $200 \mathrm{~nm}$ thick $\mathrm{Nb}$ film was then grown in a UHV chamber at room temperature. No RF plasma clean was used prior to deposition of the $\mathrm{Nb}$. To maximize the effect of the two-level systems (TLSs) in the dielectric material [8] and avoid trapped magnetic flux in the CPW line $[8,14]$, we chose a center line width of $3 \mu \mathrm{m}$ and a gap width of $2 \mu \mathrm{m}$, as described previously $[6,8,12]$.

Fourteen resonators were frequency multiplexed with seven on each half of a die separated by the microwave feedline. Resonators were divided in two halves on a die in order to distinguish between covered and uncovered devices. Figure 2 (a) shows the CPW resonators on either side of the microwave feedline. As shown in Fig. 2 (a), the coupling capacitors had no deposited materials. This sample has $300 \mathrm{~nm}$ of $\mathrm{BN}$ covering all the resonators above the feedline.

The microwave resonators had a resonance frequency in the range of $4.5 \mathrm{GHz}$ to $9.0 \mathrm{GHz}$. The $\mathrm{CPW}$ resonators were designed in pairs so that the resonance frequencies of the pair were separated by $50 \mathrm{MHz}$. Each pair was separated from the other pairs of CPWs by at least $500 \mathrm{MHz}$. This was done so that individual resonators could be easily distinguished. The CPW resonators had a coupling quality factor $\mathrm{Q}_{c}$ of 175,000 to
500,000 . In this study, a careful measurement of the kinetic inductance was not made. This lead to higher uncertainty in the calculations of the effective dielectric constant and the filling factors. For the resonators with $\mathrm{BN}$ and $\mathrm{SiO}_{2}$ deposited on the surface, the shift in resonance frequency from the theoretical value for an uncovered resonator was less than $2 \%$, meaning they had a small kinetic inductance fraction.

For the samples with $\mathrm{B}_{4} \mathrm{C}$ deposited, we found that the measured resonance frequency deviated from the theoretical value for an uncovered resonator by almost $10 \%$. With a high kinetic inductance, the effective dielectric constant reported has a high uncertainty. Depositing dielectric material on the resonators increased the effective dielectric constant, which shifted the resonance frequency lower, typically by a few hundred megahertz. For example, one resonator covered by 250 $\mathrm{nm}$ of $\mathrm{B}_{4} \mathrm{C}$ had a resonance frequency $\left(f_{r}\right)$ of $5.23 \mathrm{GHz}$, whereas a bare $\mathrm{Nb}$ resonator had a designed resonance frequency of $f_{r}=5.65 \mathrm{GHz}$.

\subsection{Dielectric Deposition}

Two different thicknesses of dielectric films for the $\mathrm{SiO}_{x}$ and the BN sample were used in this study, as listed in Table 1. For the $\mathrm{B}_{4} \mathrm{C}$ sample, only one thickness was used, $250 \mathrm{~nm}$, due to difficulties growing thicker films. The BN target had a purity of $99.99 \%$. The BN film was grown with argon $(\mathrm{Ar})$ and nitrogen $(\mathrm{N})$ gas flowing into the chamber during sputter with a rate of $15 \mathrm{sccm}$ for $\mathrm{N}$ and $\mathrm{Ar}$ at a pressure of $0.4 \mathrm{~Pa}$. The sample was at room temperature. Both thin films of $\mathrm{B}_{4} \mathrm{C}$ and $\mathrm{BN}$ were grown onto the niobium coplanar waveguides described previously in this paper.

The thin film of $\mathrm{SiO}_{2}$ was grown using a commercially available electron cyclotron resonance (ECR) machine at low power. A shadow mask was machined out of molybdenum (Mo) that enclosed the sample with an opening cut in it so that $\mathrm{B}_{4} \mathrm{C}, \mathrm{BN}$, and $\mathrm{SiO}_{2}$ would cover half the resonators (see Fig. 2). Wire-bond pads, the microwave feedline, and half the resonators on the other side of the die were completely covered to ensure material was only deposited where intended. The opening in the shadow mask was $350 \mu \mathrm{m}$ away from the transmission line. The uncovered resonators were used as control devices. This is useful for monitoring the dielectric constant of the substrate and material properties of the superconducting metal. The dielectric loss of each device was measured for covered and uncovered devices. Prior to deposition, bare $\mathrm{Nb}$ devices had a low power low temperature loss of approximately $8 \times 10^{-6}$. This ensured the resonators were not destroyed during processing and that measurements were consistent. 




Fig. 3 Inverse of the internal quality factor for niobium resonators with no deposited material heated in a low oxygen environment. The inset shows two bare $\mathrm{Nb}$ resonators on intrinsic $\mathrm{Si}(100)$. The left resonator in the inset had no annealing, while the right resonator was annealed at $300^{\circ} \mathrm{C}$.

The internal dielectric loss of the deposited material on resonators could then be obtained.

Thin films of $\mathrm{B}_{4} \mathrm{C}, 250 \mathrm{~nm}$ in thickness, were deposited by magnetron sputtering from a hot pressed $\mathrm{B}_{4} \mathrm{C}$ target with argon process gas at substrate temperatures of $20^{\circ} \mathrm{C}$ and $350^{\circ} \mathrm{C}$. The $102 \mathrm{~mm}$ target was manufactured with an epoxy bonded copper backing plate. During deposition, the target power was kept to $50 \mathrm{~W}$ and ramped at $1 \mathrm{~W} / \mathrm{min}$ to prevent cracking, yielding a deposition rate of $0.9 \AA / \mathrm{min}$. X-ray photoemission demonstrated argon and oxygen incorporation at less than $5 \%$ of the $\mathrm{B}_{3.9} \mathrm{C}$ stoichiometry for both samples.

We found $\mathrm{Nb}$ resonators with $\mathrm{B}_{4} \mathrm{C}$ deposited at 350 ${ }^{\circ} \mathrm{C}$ had a quality factor that was to low too be measured. One possible reason for the decrease in quality factor could be oxidation of the $\mathrm{Nb}$ in the resonator. Previous studies showed that the surface of $\mathrm{Nb} 3 \mathrm{~d} R \mathrm{RF}$ cavities begins to oxidize above $100^{\circ} \mathrm{C}$ and decreases the performance [20-23]. We also found that as $\mathrm{Nb}$ resonators were heated in a chemical vapor deposition (CVD) system there was an increase in the dielectric loss as shown in Fig. 3. Prior to heating, the CVD chamber was pumped for 20 minutes using a mechanical roughing pump, then the CVD chamber was purged for 10 minutes with Ar gas (600 sccm) while being pumped. The CVD was then brought to the various temperatures within 20 minutes and kept at those temperatures for an additional 30 minutes.

During heating, the CVD was evacuated using a mechanical roughing pump. For the samples heated at room temperature and at $150{ }^{\circ} \mathrm{C}$, there was no change in the dielectric loss of the device as shown in Fig. 3. For samples heated to $250^{\circ} \mathrm{C}$, the dielectric loss increases to over $10^{-5}$ as shown in Fig. 3. The inset of Fig. 3 also shows a distinct change in the color of the $\mathrm{Nb}$ for a resonator annealed at $300^{\circ} \mathrm{C}$. The pristine resonator on the left in the inset had a distinctly golden silver hue, while the resonator that was annealed at $300^{\circ} \mathrm{C}$ on the right had a dark reddish brown hue. What this shows is that when $\mathrm{Nb}$ microwave resonators are heated above $150^{\circ} \mathrm{C}$, there is a change in the $\mathrm{Nb}$ and an increase in internal loss. Most likely, this is due to oxidation of the $\mathrm{Nb}$, as reported previously [20-24]. Careful measurement of the critical temperature $\left(\mathrm{T}_{c}\right)$ of the devices shown in Fig. 3 were not made. However the changes in the $\mathrm{T}_{c}$ of $\mathrm{Nb}$ after heating were previously reported [24]. Prior to deposition of amorphous silicon (a-Si) using PECVD when $\mathrm{Nb}$ was heated to $250^{\circ} \mathrm{C}$, they measured a decrease in $\mathrm{T}_{c}$. This can be attributed to the fact that oxygen and hydrogen migrate from the surface to the bulk of the $\mathrm{Nb}$ and cause an expansion of the crystal lattice. The findings in this paper compliment the earlier work [24] and show that not only does heating $\mathrm{Nb}$ cause a structural change in $\mathrm{Nb}$ and a decrease in the $\mathrm{T}_{c}$, but it also leads to an increase in internal loss of the device.

For bare $\mathrm{Nb}$ resonators heated to $250^{\circ} \mathrm{C}$, there was a shift in the resonance frequency by about $2 \mathrm{GHz}$. Whereas, the sample heated to $150^{\circ}$ had only a slight shift to a lower resonance frequency of less than 100 $\mathrm{MHz}$. Since the device geometry hasn't changed, either the dielectric material under the metal changed or the metal itself changed.

\section{Power Dependence of Loss}

Fig. 4 shows the loss tangent of two different samples of $\mathrm{B}_{4} \mathrm{C}(250 \mathrm{~nm})$ and a bare $\mathrm{Nb}$ resonator. The data points for the two samples are shown as circles and squares. The power fit of the data is shown as a dashed lined using Eq. 2. We find that $\delta_{i}$ for the uncovered $\mathrm{Nb}$ resonator was $8.2 \times 10^{-6}$. This shows that the dielectric films were effectively masked. Also, Auger electron spectroscopy (AES) showed no evidence of the deposited dielectric on the covered side of the wafer. However, there was a change in resonance frequency with a corresponding change in the effective dielectric constant as calculated with Eq. 3. Deviations in the dielectric constant can be used as an indicator of issues with the device as a whole.

The intrinsic loss due to two level systems, $\delta_{t l s}^{0}$, was measured using the internal loss vs. the power. In order to find $\delta_{t l s}^{0}$, the internal quality factor, $Q_{i}$, must first 


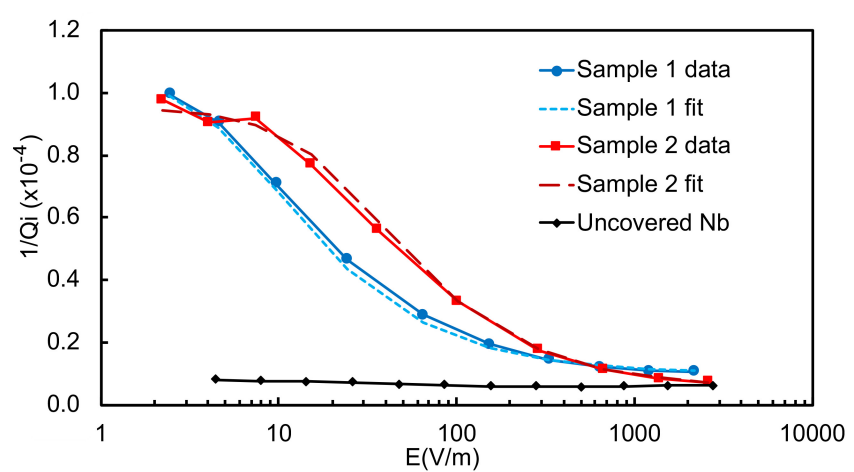

Fig. 4 Internal loss of two resonators with deposited $\mathrm{B}_{4} \mathrm{C}$ and one bare $\mathrm{Nb}$ resonator on sapphire. Sample 1 and 2 both had $\mathrm{B}_{4} \mathrm{C}$ grown at room temperature. The loss tangents for both samples with deposited $\mathrm{B}_{4} \mathrm{C}$ appear to converge to the same value for low power. Uncovered $\mathrm{Nb}$ on sapphire is shown in black for comparison.

be found. For a resonator, $Q_{i}$, can be found by solving the equation

$$
\frac{1}{Q_{m}}=\frac{1}{Q_{c}}+\frac{1}{Q_{i}} \text {. }
$$

where $Q_{m}$ is the measured quality factor and $Q_{c}$ is the coupling quality factor. Both $Q_{m}$ and $Q_{c}$ were extracted from the measured transmission $S_{21}$ data using the circle fit method $[6,12,17]$. A microwave electromagnetic simulator was also used to calculate $Q_{c}[6]$ based on the method of Ben Mazin [25]. The measured values of $Q_{c}$ obtained from the circle fit method were lower than the designed values by up to $33 \%$. This discrepancy is well known and could be due to the proximity to other resonators or other factors introduced during fabrication [25].

Internal loss, $1 / Q_{i}$, as a function of electric field strength is shown in Fig. 4. Eq. 1 is used to separate the internal quality factor from the measured quality factor. Internal loss $\delta_{i}=1 / Q_{i}$ is composed of two terms, $\delta_{i}=\delta_{t l s}^{0}+\delta_{0}$, where $\delta_{t l s}^{0}$ is the power dependent loss due to two-level systems, and $\delta_{0}$ is the background loss, which is power independent [18]. The internal loss is fit in Fig. 4 using the equation

$$
\frac{1}{Q_{i}}=\frac{F \delta_{t l s}^{0} \tanh \left(\frac{\hbar \omega}{2 k_{B} T}\right)}{\sqrt{1+\left(\frac{E}{E_{c}}\right)^{\beta}}}+\delta_{0}
$$

where $F$ is the filling factor of the dielectric in the CPW gap, $\hbar$ is Planck's constant, $k_{B}$ is Boltzman's constant, $E$ is the power in the resonator, and $E_{c}$ is a fitting paramterter associated with the critical power above which the TLSs are "saturated" [16]. Figure 4 shows that there is good agreement between measured internal quality factor data, circles and squares, and the fitting function Eq. 1, dashed lines. We found that the two samples of $\mathrm{B}_{4} \mathrm{C}$ had different values of $E_{c}$ using
Table 1 Comparison of internal loss at low power and temperature using $F \delta_{\text {TLS }}^{0}$ using Eq. 4 and Eq. 2. The first column is the deposited material, the second is the thickness of the deposited material on $\mathrm{Nb}, \delta_{i}$ is the measured internal loss, $\varepsilon_{\text {dep }}$ is the dielectric constant of the deposited material, $F$ is the filling factor, and $F \delta_{\text {TLS }}^{0}$ temp is the intrinsic loss due to TLSs times the filling factor taken from the temperature dependent data. The column $F \delta_{\text {TLS }}^{0}$ power was taken from a power fit of the data. Parentheses indicate the uncertainty in the last digit.

\begin{tabular}{llllll}
\hline Material & thickness $(\mathrm{nm})$ & $\delta_{i}\left(\times 10^{-4}\right)$ & $\varepsilon_{\text {dep }}$ & $F$ & $\begin{array}{l}F \delta_{\mathrm{TLS}}^{0} \\
\text { temp } \\
\left(\times 10^{-4}\right)\end{array}$ \\
\hline $\mathrm{B}_{4} \mathrm{C} / \mathrm{Nb}$ & $250(10)$ & 1.0 & $4(2)$ & $0.04(2)$ & $1.50(4)$ \\
$\mathrm{BN} / \mathrm{Nb}$ & $170(5)$ & 0.23 & $3.2(5)$ & $0.019(4)$ & $0.35(5)$ \\
$\mathrm{BN} / \mathrm{Nb}$ & $300(5)$ & 0.516 & $3.5(3)$ & $0.034(4)$ & $0.57(1)$ \\
$\mathrm{SiO}_{2} / \mathrm{Nb}$ & $450(5)$ & 1.73 & $3.5(9)$ & $0.05(2)$ & $2.02(1)$ \\
$\mathrm{SiO}_{2} / \mathrm{Nb}$ & $900(5)$ & 2.20 & $3.4(9)$ & $0.08(2)$ & $4.00(1)$
\end{tabular}

Eq. 1: 7 for sample 1 and 25 for sample 2 shown in Fig.4. This difference in value could be due to variations in the deposition of $\mathrm{B}_{4} \mathrm{C}$. Variation in sample fabrication is unfortunately a much bigger issue that plagues many devices in quantum information. To realize a quantum computer, reproducibility among devices will be a key in large scale arrays of qubits. The filling factor was calculated by taking the capacitance in the region with the deposited dielectric material, divided by the total capacitance of the device. The results for this method of fitting for all materials in this study are listed in Table 1. Capacitance for each region (the substrate, sputter deposited material and vacuum) were calculated according to the references $[7,19]$. When calculating the capacitance of a device, it is necessary to know the effective dielectric constant, $\varepsilon_{e f f}$. We found $\varepsilon_{\text {eff }}$ using the equation

$\varepsilon_{e f f}=\frac{c}{4 l} \frac{1}{f_{r}}$

where $c$ is the speed of light, $l$ is the physical length of the resonator, and $\mathrm{f}_{r}$ is the resonance frequency of the $\mathrm{CPW}$. The length of the resonators $l$ was known from the design of the CPW. The dielectric constant of the material, $\varepsilon_{r}$, was determined using $\varepsilon_{e f f}$ and conformal mapping described by Simmons [19].

The intrinsic dielectric loss tangent $\delta_{t l s}^{0}$, the dashed lines shown in Fig. 4, can be extracted from Eq. (2) by fitting the data for $1 / \mathrm{Q}_{i}$ according to the references $[1,16,18]$. The value of the exponent $\beta=1.6$, was the best value for fitting the data in agreement with the literature $[1,18]$. 


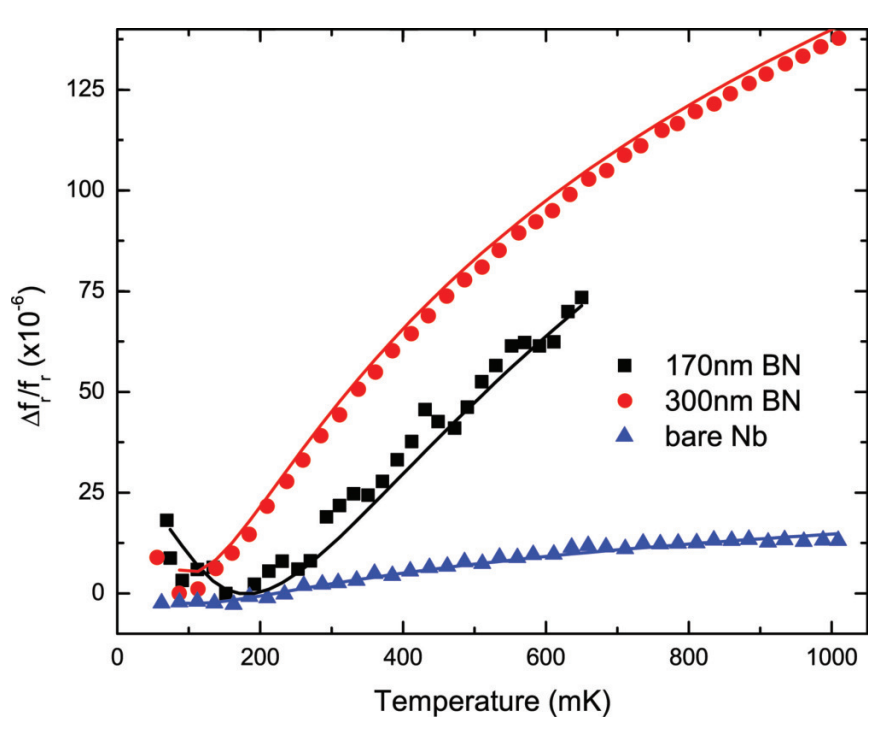

Fig. 5 The relative resonance frequency vs temperature shift for $\mathrm{BN}$ is shown. Dielectric loss was measured for the deposited $\mathrm{BN}$ as described in the experimental setup and shown in the figure. Shapes represent the actual data points while the solid lines are fits of the data using Eq. 4.

\section{Temperature Dependence of Loss}

The zero-temperature loss due to two-level systems, $\delta_{t l s}^{0}$ can be measured at a fixed high power, typically -70 $\mathrm{dBm}$ or an electric field strength of $\mathrm{E} \approx 8000 \mathrm{~V} / \mathrm{m}$, using the temperature dependence of the resonance frequency,

$$
\frac{\Delta f_{r}(T)}{f_{r}}=F \delta_{t l s}^{0}\left[\Re \Psi\left(\frac{1}{2}-\frac{\hbar \omega}{2 i k_{B} T}\right)-\log \left(\frac{\hbar \omega}{2 \pi k_{B} T}\right)\right]
$$

where $\Delta f_{r}$ is the frequency shift, $f_{r}$ is the resonance frequency at low temperature, $\omega$ is the angular frequency $2 \pi f, T$ is the temperature, and $\Re \Psi$ is the real part of the digamma function. Fitting using equation 4 was taken from ref. [6]. For consistency, $\delta_{t l s}^{0}$ from the temperature dependent data was checked and found to be consistent with that found from the power dependent data as shown in Table 1. In Fig. 5 the measured relative resonance frequency as a function of temperature is fit using Eq. 4 for samples covered with BN.

Extracting the internal dielectric loss, $\delta_{t l s}^{0}$, using Eq. 4 is much easier than using the power fit method since low power scans are not required, as discussed in the literature [6].

\section{Results and Discussion}

Using Eq. 4 to fit the frequency versus temperature data, values for $F \delta_{t l s}^{0}$ are given in Table 1 . We found that $\mathrm{B}_{4} \mathrm{C}$ has a zero-temperature TLS loss of $F \delta_{t l s}^{0}$ of between $1 \times 10^{-4}$ and $1.5 \times 10^{-4}$, and $\mathrm{BN}$ has a zerotemperature TLS loss of $F \delta_{t l s}^{0}=0.60 \times 10^{-4}$ as shown in
Table 1. Both of these two materials had a lower zerotemperature TLS loss than that of $\mathrm{SiO}_{2}$ by roughly a factor of 2 to 3 . We also found that as the thickness of the $\mathrm{BN}$ and $\mathrm{SiO}_{x}$ increased, $F \delta_{t l s}^{0}$ increased as well. For $\mathrm{BN}$ and $\mathrm{SiO}_{x}$, if $F \delta_{t l s}^{0}$ is divided by $F$, then one finds a constant value of $\delta_{t l s}^{0}=1.7(1) \times 10^{-3}$ and $\delta_{t l s}^{0}=4.3(2) \times 10^{-3}$ respectively (the parentheses indicate the uncertainty in the last digit), implying that TLSs are distributed throughout the amorphous dielectric layer as listed in Table 1.

The main mechanism known that causes dielectric loss at low temperature and low power is two-levels systems [29-31]. Two-level systems (TLSs) have an electric dipole and are associated with dangling bonds. For example, in $\mathrm{SiO}_{2}$ and $\mathrm{SiN}_{x}$, the loss is attributed to the strong polar microwave absorption from molecules such as $\mathrm{OH}^{-}$or other impurities with an electric dipole moment such as $\mathrm{F}^{-}$and $\mathrm{Cl}^{-}$atoms. Paik et al. [10] observed a correlation between the number of $\mathrm{NH}_{2}$ molecules and the dielectric loss in their $\mathrm{SiN}_{x}$ films. These impurities act like two-level systems that become unsaturated at low temperature and power and thus begin absorbing power. Polar molecules like $\mathrm{NH}_{2}$ that are present in SiN due to the growth process, provide a clear physical example of how a two-level system could absorb power from a microwave circuit. As the electric field of the microwave acts on the dipole moment of these molecules, it can absorb the microwave photon and be excited into a rotational or vibrational state. The molecule has a certain lifetime in the excited state and then will decay via microwave photons or phonons.

For $\mathrm{B}_{4} \mathrm{C}$ and $\mathrm{BN}$, a considerable numbers of defects are known to be distributed throughout magnetron sputtered films depending on the growth conditions. Werheit et al. $[32,33]$ point out that $\mathrm{B}_{4} \mathrm{C}$ contains a high level of substitutional disorder, thereby creating electronic defects in the material. These defects act as dopants, i.e. electric dipoles, as reported by Schmechel et al. [34]. Due to the amorphous nature of the magnetron sputtered $\mathrm{BN}$ and $\mathrm{B}_{4} \mathrm{C}$, there is a broad spectrum of energy activation of these defects; thus, these defects can act as the two level systems that can absorb energy at low power and low temperature in the RF regime. With respect to the materials presented in this study, it means that if defects could be removed from $\mathrm{B}_{4} \mathrm{C}$ and $\mathrm{BN}$, the loss in these dielectric materials could be removed.

This work also speaks to a bigger challenge in device fabrication [26]. Growing crystalline Josephson junctions in a qubit has been a goal of many research groups over the years. This was successful to a certain extent [27], but did not completely solve the problem of TLSs in barriers. What this work shows is that the fabrication process in producing crystalline materials could be 
causing other issues in the devices. Most likely, producing the crystalline element of a device, such as a Josephson junction in a qubit, will require annealing the entire device. Since annealing a device increases the likelihood of oxidation of metallic layers, this could be problematic for incorporating crystalline materials in devices for quantum computing. Recently, Kumar et al. [28] found strong evidence that molecular oxygen at the superconducting metal surface can play a large role in unwanted noise in superconducting devices. One important question then is whether or not molecular oxygen incorporated into a material, such as sputter-deposited $\mathrm{B}_{4} \mathrm{C}$, could also lead to increased internal loss.

This research shows further studies on $\mathrm{B}_{4} \mathrm{C}$ and $\mathrm{BN}$ are warranted since these materials have variable mechanical and electronic properties that depend on their growth conditions $[5,35]$. As with $\mathrm{SiO}_{2}$ and $\mathrm{SiN}_{x}$, it may be possible to improve the loss for $\mathrm{B}_{4} \mathrm{C}$ and $\mathrm{BN}$ dielectrics by altering the sputter deposition recipe. Neither of these materials have had extensive studies done on the dielectric loss in the context of devices for quantum computing. One possibility would be to vary the sputter deposition conditions with which $\mathrm{B}_{4} \mathrm{C}$ and $\mathrm{BN}$ are grown. Alternatively, using a different superconducting material such as aluminum, and annealing the devices during or after deposition could remove impurities and increase order in the deposited materials. However, in the case of $\mathrm{BN}$, if the substrate is heated, it is known that BN will not stick to the surface. The datasets generated during and/or analyzed during the current study are available from the corresponding author on reasonable request.

\section{Conclusion}

In conclusion, we have shown $\mathrm{B}_{4} \mathrm{C}$ has a zero-temperature TLS loss of $F \delta_{t l s}^{0}$ of between $1 \times 10^{-4}$ and $1.5 \times 10^{-4}$, while boron nitride has a zero-temperature TLS loss of $F \delta_{t l s}^{0}=0.60 \times 10^{-4}$ which are both lower than that of $\mathrm{SiO}_{2}$ by roughly a factor of 2 to 3 . Boron nitride $(\mathrm{BN})$ and boron carbide $\left(\mathrm{B}_{4} \mathrm{C}\right)$ are straightforward to grow using sputter deposition which makes them very attractive for a wiring crossover. We also found that deposition of dielectric materials at elevated temperatures, above $150^{\circ} \mathrm{C}$, can have unwanted effects on the circuit as a whole such as oxidation of the superconducting metal, a decrease in quality factor, and a change in resonance frequency of the devices.

\section{References}

1. J. M. Sage, V. Bolkhovsky, W. D. Oliver, B. Turek, P. B. Welander, J. Appl. Phys. 109,063915 (2011)
2. V. L. Solozhenko, N. A. Dubrovinskaia, L. S. Dubrovinsky, Appl. Phys. Lett. 85, 1508, (2004)

3. R. Sousa, Phys. Rev. B 76, 245306 (2007)

4. F. Yoshihara, Y. Nakamura, J. S. Tsai, Phys. Rev. B 81, $132502(2010)$

5. M. D. Wiggins, C. R. Aita, J. Vac. Sci. Tech. 2, 322 (1984)

6. J. Gao, Caltech Ph.D. thesis (2008)

7. A. D. O'Connell, M. Ansmann, R. C. Bialczak, M. Hofheinz, N. Katz, E. Lucero, C. McKenney, M. Neeley, H. Wang, E. M. Weig, A. N. Cleland, and J. M. Martinis, Appl. Phys. Lett. 92, 112903 (2008)

8. J. Gao, M. Daal, A. Vayonakis, S. Kumar, J. Zmuidzinas, B. Sadoulet, B. A. Mazin, P. K. Day, H. G. Leduc, Appl. Phys. Lett. 92, 152505 (2008)

9. R. Barends. H. L. Hortensius, T. Zijlstra, J. J. A. Baselmans, S. J. C. Yates, J. R. Gao, T. M. Klapwijk, Appl. Phys. Lett. 92, 223502 (2008)

10. H. Paik, K. D. Osborn, Appl. Phys. Lett. 96, 072505 (2010)

11. A. Brunoa, S.T. Skacelc, Ch. Kaiserc, S. Wu?nschc, M. Siegelc, A.V. Ustinovd, M.P. Lisitskiya, Physics Procedia textbf36, 245 ? 249 (2012)

12. D. Wisbey, J. Gao, M. Vissers, Fabio C. S. da Silva, J. S. Kline, L. Vale, D. Pappas, J. Appl. Phys. 108, 093918 (2010)

13. A. Megrant, C. Neill, R. Barends, B. Chiaro, Y. Chen, L. Feigl, J. Kelly, E. Lucero, M. Mariantoni, P. O'Malley, D. Sank, A. Vainsencher, J. Wenner, T. White, Y. Yin, J. Zhao, C. Palmstr $\phi \mathrm{m}$, J. Martinis, A. Cleland, Appl. Phys. Lett. 100, 113510 (2012)

14. C. Song, T. W. Heitmann, M. P. DeFeo, K. Yu, R. McDermott, M. Neeley, J. M. Martinis, B. L. T. Plourde, Phys. Rev. B 79, 174512 (2009)

15. M. Göppl, A. Fragner, M. Baur, R. Bianchetti, S. Filipp, J. M. Fink, P. J. Leek, J. Appl. Phys. 104, 113904 (2008)

16. D. P. Pappas, M. R. Vissers, D. S. Wisbey, J. S. Kline, J. Gao, IEEE Trans. Appl. Supercond. 21, 871 (2011)

17. S. Probst, F. B. Song, P. A. Bushev, A. V. Ustinov, and M. Weides Rev. Sci. Instrum. 86, 024706 (2015)

18. H. Wang, M. Hofheinz, J. Wenner, M. Ansmann, R. Bialczak, M. Lenander, E. Lucero, M. Neeley, A. O'Connell, D. Sank, M. Weides, A. Cleland, and John Martinis, Appl. Phys. Lett. 95, 233508 (2009)

19. R. N. Simons, "Coplanar Waveguide Circuits, Components, and Systems" Willey Series in Microwave and Optical Engineering, Kai Chang, Series Editor (2001) By John Wiley and Sons, ISBN 0-471-16121-7

20. O. Hellwig, H. Zabel, Physica B, 283, 228 (2000)

21. O. Hellwig, H. W. Becker, H. Zabel, Phys. Rev. B, 64, 233404 (2001)

22. T. Proslier, J. Zasadzinski, J. Moore, M. Pellin, J. Elam, L. Cooley, C. Antoine, J. Norem, K. E. Gray, Appl. Phys. Lett. 93, 192504 (2008)

23. J. Halbritter, Appl. Phys. A 43, 1-28 (1987)

24. A. Bruno, P. Mengucci, L. V. Mercaldo, M. P. Lisitskiy, Phys. Procedia 36, 239-244, (2012)

25. B. Mazin, Caltech Ph.D. thesis (2008)

26. S. Oh, K. Cicak, J. S. Kline, M. A. Sillanp, K. D. Osborn, J. D. Whittaker, R. W. Simmonds, D. P. Pappas, Phys. Rev. B 74, 100502(R) (2006)

27. M. Weides, J. S. Kline, M. Vissers, M. Sandberg, D. S. Wisbey, B. R. Johnson, T. Ohki, and D. P. Pappas, Appl. Phys. Lett. 99, 262502 (2011)

28. P. Kumar, S. Sendelbach, M. A. Beck, J. W. Freeland, Zhe Wang, H. Wang, C. C. Yu, R. Q. Wu, D. P. Pappas, R. McDermott, Phys. Rev. Appl. 6, 041001 (2016)

29. W. A. Phillips, Rep. Prog. Phys. 50, 1657 (1987) 
30. M. Von Schickfus, S. Hunklinger, Phys. Lett. 64, 144 (1977)

31. M. Von Schichfus, S. Hunklinger, J. Phys. C: Solid State Phys. 9, L439 (1976)

32. H. Werheit, H. W. Rotter, F. D. Meyer, H. Hillebrecht, S.

O. Shalamberdze, T. G. Abzianidze, G. G. Esadze, J. Solid State Chem. 177, 569 (2004)

33. H. Werheit, T. Au, R. Schmechel, J. Solid State Chem. 154, 79 (1999)

34. R. Schmechel, H. Werheit, J. Phys.: Condens. Matter 11, 6803 (1999)

35. L. G. Jacobsohn, M. Nastasi, Surf. Coat. Technol. 200, $1472(2005)$ 